\title{
A qualidade da assistência pré-natal sob olhar multiprofissional
}

\author{
The quality of pre-natal assistance under a multiprofessional perspective
}

\author{
Bárbara Maldonado Tomazettia, Letícia Hermes ${ }^{\mathrm{b}}$, Naiashy Vanuzzi Martelloc, \\ Patrícia Menezes Schmittd, Melissa Medeiros Braze, Izabel Cristina Hoffmann ${ }^{f}$ \\ Nutricionista. Especialista em Gestão e Atenção Hospitalar no Sistema Público de Saúde pela Universidade Federal de Santa Maria (UFSM).

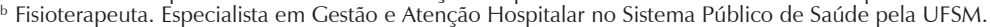 \\ c Enfermeira. Especialista em Gestão e Atenção Hospitalar no Sistema Público de Saúde pela UFSM. \\ d Terapeuta Ocupacional. Especialista em Gestão e Atenção Hospitalar no Sistema Público de Saúde pela UFSM. \\ e Fisioterapeuta. Doutora em Engenharia de Produção pela Universidade Federal de Santa Catarina. Professora do Curso de Fisioterapia da UFSM. \\ Enfermeira. Doutora em Ciências. Atua no Hospital Universitário de Santa Maria. Tutora de Campo e Núcleo do Programa de Residência Multiprofissional \\ em Gestão e Atenção Hospitalar no Sistema Público de Saúde da UFSM.
}

RESUMO

Objetivo: Avaliar a qualidade da assistência pré-natal, sob a ótica dos profissionais de saúde das Estratégias de Saúde da Família (ESF) do município de Santa Maria (RS).

Materiais e Métodos: Constitui-se como uma pesquisa de campo, do tipo descritiva e com abordagem qualitativa, realizada em 13 ESF do município, entre junho e julho de 2015. Para a coleta dos dados, foi utilizado uma entrevista semiestruturada e a análise de conteúdo.

Resultados: Elencou-se 5 categorias temáticas: Acolhimento e acompanhamento das gestantes nas Estratégias de Saúde da Família; Estratégias de intervenção na atenção à gestante de baixo risco; Rotinas e limites na realização de exames e procedimentos obstétricos; Atuação multiprofissional e seus diferentes papéis no atendimento Pré-Natal; Encaminhamento das gestantes para os serviços de referência e contra referência.

Conclusão: Verificou-se a necessidade de qualificar os processos de trabalho na assistência pré-natal, com estratégias eficazes a fim de evitar práticas fragmentadas que provocam distanciamento entre serviços/profissional-gestante, garantindo a integralidade do cuidado em todos os níveis de atenção.

Palavras-chave: assistência pré-natal; estratégia saúde da família; qualidade da assistência à saúde.

ABSTRACT I Objective: This study aims to evaluate the quality of prenatal care, through the eyes of health professionals working on the Strategies for Family Health program (ESF) from the city of Santa Maria, Brazil.

Materials and Methods: It is a field research (descriptive), with a qualitative approach, including 13 ESF, between June and July of 2015. For the data collection, semi-structured interviews were conducted, followed by content analysis.

Results: In these interviews, 5 subject categories were assessed: Pregnant reception and follow-up in the Strategies for Family Health program; Intervention strategies for the care of low-risk pregnancy; Routines and limits on the obstetric exams and procedures; Multiprofessional work and its different roles in the prenatal care; Referral of pregnant women to referral and counter-referral services. Conclusion: It is necessary to qualify the work processes in the prenatal care, using effective strategies in order to avoid fragmented practices that increase the distance between professionals/services and the pregnant, ensuring the wholeness of the care in all levels.

Keywords: prenatal care; family health strategy; quality of health care. 


\section{INTRODUÇÃO}

A assistência pré-natal tem como finalidade acolher a mulher desde o momento em que a mesma evidenciou a sua gestação, e cabe ao profissional de saúde oferecer atenção qualificada e integral às gestantes, garantindo um cuidado mais humanizado, além de tal prática ser importante para a redução da mortalidade materna e infantil' ${ }^{1}$.

A atenção básica, especialmente a Estratégia de Saúde da Família (ESF), visa à promoção da saúde materna, prevenção e tratamento de complicações que possam ocorrer no período gravídico e puerperal ${ }^{2}$. As ESFs possuem uma particularidade centrada no cuidado, na escuta qualificada e no vínculo entre profissional-paciente. A atenção à gestante e o cuidado pré-natal são características específicas do processo de trabalho das ESFs, pois conferem maior proximidade com as gestantes, especialmente pela atuação dos Agentes Comunitários de Saúde, que prestam assistência e fazem parte da equipe mínima preconizada pelo Ministério da Saúde ${ }^{3,4}$.

A integração entre diversos profissionais da saúde nas ESFs possibilita diferentes olhares sobre as práticas do cuidado à saúde materno-infantil, garantindo uma atenção integral e aumentando o potencial de resolutividade ${ }^{5}$. Dentro do campo de atuação da atenção materno-infantil, apesar dos avanços, a assistência ao pré-natal no Brasil ainda é um fator preocupante na saúde pública e requer uma atenção especial ${ }^{1}$. Estudos demonstram que a ausência de assistência pré-natal está associada à maior taxa de mortalidade perinatal ${ }^{2}$.

A lacuna da assistência integral no pré-natal gera um impacto no desfecho perinatal, devido às gestantes chegarem muitas vezes desinformadas no momento do parto. Dentre as ações realizadas durante a consulta pré-natal, as orientações às gestantes são essenciais para prepará-las para todo o processo gestacional, parto e pós-parto ${ }^{6}$. Diante disso, o Ministério da Saúde (MS) oferece aos profissionais que prestam assistência ao pré-natal, protocolos de procedimentos e condutas a serem realizadas durante todo o pré-natal a fim de garantir a qualidade em toda rede de atenção à gestante 7 .

Diante da relevância da temática o presente estudo teve por objetivo avaliar, sob a ótica dos profissionais de saúde das ESFs, a qualidade da assistência pré-natal do município de Santa Maria, RS.

\section{MATERIAIS E MÉTODOS}

O presente estudo constitui-se como uma pesquisa de campo, do tipo descritiva e com abordagem qualitativa. A coleta de dados foi realizada nas 13 ESF existentes no município de Santa Maria, RS, no período entre junho e julho de 2015.

Participaram do estudo 26 profissionais de saúde que exerciam atividades junto às gestantes durante 0 acompanhamento pré-natal. Dentre esses profissionais, 5 Médicos, 12 Enfermeiros, 4 Técnicos em Enfermagem e 5 Agentes Comunitários de Saúde (ACS). Em cada ESF, foram entrevistados pelo menos dois profissionais da equipe mínima preconizado pelo MS de núcleos distintos, visando à imparcialidade frente à atividade que desempenhavam junto às gestantes em acompanhamento de pré-natal ${ }^{8}$.

Foram incluídos neste estudo os médicos que fazem parte do "Programa Mais Médicos" e são provenientes de outros países, além de profissionais do Programa de Valorização dos Profissionais da Atenção Básica - PROVAB, que atuam na ESF num período menor que 01 ano. Ainda, participaram profissionais residentes do Programa de Residência Multiprofissional em Saúde da Universidade Federal de Santa Maria (UFSM). É importante ressaltar que o anonimato dos participantes foi viabilizado com a utilização do sistema alfanumérico de representação dos dados com as letras " $\mathrm{Pr}$ " (de profissional) e os números distribuídos de forma aleatória.

Para atender os aspectos éticos, foram seguidas as recomendações da Resolução CNS no 466/12, que envolve a pesquisa com seres humanos, respeitando a normativa do serviço, além do sigilo e integridade dos sujeitos envolvidos ${ }^{9}$. Esse artigo é um dos produtos da pesquisa desenvolvida no Programa de Residência Multiprofissional Integrada em Gestão e Atenção Hospitalar no Sistema Único de Saúde da Universidade Federal de Santa Maria, intitulada 'A qualidade da assistência pré-natal e seu reflexo no desfecho hospitalar sob a ótica de puérperas e profissionais da saúde do município de Santa Maria, $R S^{\prime}$. O projeto foi registrado e aprovado pelo Comitê de Ética em Pesquisa da UFSM sob parecer número 1.091.063 em 09/06/2015.

Os dados foram coletados somente após a autorização e assinatura do Termo de Consentimento Livre e Esclarecido pelos participantes; foi utilizado como recurso técnico uma entrevista semiestruturada constituída por 11 questões, gravadas e transcritas pelas pesquisadoras para análise. Posteriormente, iniciou-se a fase de análise de conteúdo, por meio da leitura das transcrições provenientes das falas dos sujeitos entrevistados ${ }^{10}$.

Considerou-se a análise de conteúdo como método de organização e análise de dados qualitativos, examinou-se os elementos constitutivos para compreender e explicar a dinâmica das relações sociais vivenciadas no cotidiano dentro de estruturas e instituições ${ }^{11}$. Estudos apontam que essa escolha pode ser justificada pela necessidade de 
enriquecer a leitura e esclarecer as relações além das falas propriamente ditas ${ }^{12}$.

\section{RESULTADOS E DISCUSSÃO}

Com base na análise de conteúdo das entrevistas, foram elaboradas cinco categorias temáticas, para compreensão dos resultados obtidos.

\section{Acolhimento e acompanhamento das gestantes nas Estratégias de Saúde da Família}

O Programa de Humanização no Pré-Natal e Nascimento (PHPN) surgiu no ano de 2000 para assegurar o acesso universal da qualidade do acompanhamento pré-natal, da assistência ao parto, puerpério e ao período neonatal na perspectiva dos direitos básicos de cidadania ${ }^{13}$.

A prática do acolhimento tem como finalidade identificar as gestantes do território de abrangência da ESF e a partir disso, receber, escutar e oferecer atenção qualificada, humanizada, eficaz e segura às gestantes. $\mathrm{O}$ acolhimento baseia-se no trabalho em equipe, de forma integrada entre os mesmos e não somente num grupo de profissionais ${ }^{14}$.

Pr1 'O acolhimento não tem rotina, $o$ acolhimento deve ser feito no momento em que a pessoa precisa, então a primeira observação que a gente faz é quando a mulher tá recebendo a notícia da gestação (...)'.

$\operatorname{Pr} 3$ 'O acolhimento é feito por todos, que é principalmente acolher e escutar a queixa do paciente que pode ser sentida ou percebida, e depois tratar de orientá-la a conduta a seguir (....'.

O acolhimento é um dispositivo fundamental na política de humanização, baseado na escuta qualificada, permitindo que a gestante expresse seus medos, anseios, dúvidas e preocupações, oferecendo resolubilidade dos problemas, criando o vínculo profissional-usuária e permitindo o empoderamento destas no processo de gestação e parto ${ }^{3,15}$.

Além disso, o acolhimento é visto como um modo de reorganização dos processos de trabalho das equipes, uma vez que para acolher as usuárias com qualidade e equidade, os profissionais precisam se reunir com regularidade para debater e definir a forma como cada um irá participar nas diversas etapas do cuidado, sendo necessário que a equipe amplie sua capacidade clínica, para realizar a escuta qualificada, para que classifique os riscos e situações de vulnerabilidade, fazendo as devidas intervenções e/ou encaminhando para o serviço especializado, quando necessário ${ }^{16}$.

Por outro lado, foi observado em uma fala, um desconhecimento referente ao acolhimento da gestante.
$\operatorname{Pr} 5$ 'A princípio não. Eu nunca... não tive nenhuma participação se há ainda esse acolhimento (...)'.

Esse resultado de desconhecimento sobre acolhimento também foi encontrado em outro estudo ${ }^{17}$, no qual um profissional mostrou desconhecimento sobre o tema e confuso quando questionado. Isto denota que os profissionais nem sempre estão apropriados sobre o significado do acolhimento e que ações de educação permanente em saúde poderiam ser realizadas neste sentido, com o intuito de melhorar a qualidade dos serviços de saúde, garantindo a equidade no cuidado e aprimorando a qualidade da assistência pré-natal ${ }^{18}$.

Os profissionais de saúde precisam salientar às gestantes sobre a importância do acompanhamento pré-natal na promoção da saúde e na prevenção e tratamento de resultados desfavoráveis durante e após a gestação, garantindo-as o acesso aos serviços de saúde, bem como atenção integral e humanizada ${ }^{13}$.

Pr15 'A doutora costuma ligar, até no dia mesmo se ela se atrasou, a consulta é as duas e chegou três e ela ainda não apareceu, ela liga e depois se não, a gente faz a busca ativa no próximo dia de visita domiciliar (...)'.

Pr19 'Daí a gente tenta fazer uma busca ativa, ou ligando para o telefone, ou indo na casa em visita domiciliar, ou acionando o agente comunitário responsável pela gestante'.

Diante das falas mencionadas acima, pode-se perceber que quando as gestantes faltam às consultas previamente agendadas e/ou não procuram o serviço de saúde, os profissionais entram em contato com essas gestantes via telefone e/ou realizam a busca ativa e remarcam a consulta para continuar o acompanhamento ou iniciar o pré-natal precocemente.

A assistência pré-natal é fundamental, pois permite diagnosticar e tratar possíveis complicações durante o período gestacional. Além disso, a captação precoce da gestante possibilita identificar os riscos e encaminhá-la ao serviço de alto risco para realizar as intervenções necessárias, evitando assim, a morbimortalidade materna e perinatal ${ }^{2}$.

Conforme a Portaria 1.459, de 24 de junho de 2011, que institui a Rede Cegonha no âmbito do SUS, para assegurar a atenção humanizada à gravidez, ao parto e ao puerpério, as ações relacionadas ao componente Pré-Natal dizem respeito entre outras, à captação precoce da gestante, ao acolhimento com classificação de risco/vulnerabilidade e acesso ao prénatal de alto risco em tempo oportuno ${ }^{19}$. 
Dessa forma, para melhoria da assistência pré-natal a equipe de saúde precisa estar capacitada para resolver os principais problemas na atenção primária, realizando a captação precoce e busca ativa das gestantes que não comparecem às consultas ${ }^{2}$.

Para um adequado acompanhamento pré-natal, é recomendado realizar, no mínimo, seis consultas de prénatal $^{3}$. Um estudo apontou que $87,5 \%$ dos profissionais mencionaram a necessidade de realizarem mais de seis consultas para obter um bom acompanhamento materno fetal durante a gestação ${ }^{20}$.

Porém, nosso estudo aponta limites quando as gestantes não comparecem às consultas, visto que muitas trocam de localidade e/ou telefone para contato, sem comunicar a unidade de saúde. Além disso, existem áreas que não estão cobertas por ACS, devido à deficiência de profissionais contratados, o que dificulta a assistência às gestantes.

Pr21 'Se tem agente comunitário de saúde na micro área dela (gestante), a gente primeiro entra em contato para ele fazer a busca ativa e espera o retorno dele (ACS). Caso não tem, a gente entra em contato via telefone, mas encontramos barreiras (...) pois, muitas trocam de telefone, dá sempre desligado, (...) muitas vezes a gente não consegue sair pra fazer a visita, a gente espera que ela retorne e as vezes a gente perde essa gestante $(. . .)^{\prime}$.

A busca ativa de gestantes faltosas é uma estratégia eficaz para um bom acompanhamento pré-natal realizada por ACS. Delimitar a área de abrangência de unidades de saúde auxilia na localização precoce das gestantes, incentivando-as a comparecer nas consultas, pois a proximidade geográfica facilita o contato dos sujeitos com as unidades de saúde ${ }^{17}$.

\section{Estratégias de intervenção na atenção à gestante de baixo risco}

Os profissionais de saúde são os responsáveis por prestar uma assistência qualificada à gestante, por meio de condutas acolhedoras, proporcionando à mulher a oportunidade de expressar seus sentimentos, seja de forma individual ou em grupos, contribuindo para que as gestantes mantenham um vínculo com os profissionais de saúde durante todo o período gestacional, diminuindo os riscos de intercorrências obstétricas ${ }^{21}$.

Nesse estudo, observou-se que os profissionais ao conduzirem as consultas de pré-natal fazem uso das recomendações do protocolo do $\mathrm{MS}^{3}$.

Pr2 '(...) é feito o cadastro, é colocado num livro, agora temos uma carteira nova das gestantes (...)'.
Pr23 'A gestante chega (...) converso com ela, para ver como é que ela está, (...) se tem alguma queixa, se teve contração ou alguma intercorrência (...) fora isso, a gente faz o exame físico, peso, pressão, BCF, dinâmica, movimentação fetal (...), preenche os dados na carteirinha, se tem alguma gestante de alto risco encaminha para o pré-natal de alto risco, solicita os exames de rotina (...)'.

É preconizado que se realize na primeira consulta anamnese da gestante, exame físico completo, além de exame ginecológico e obstétrico. Bem como, levar em consideração as queixas das gestantes, a fim de esclarecer as dúvidas, medos e anseios das mesmas. Questões referentes à alimentação, movimentação fetal, perdas vaginais, entre outros, também precisam ser interrogadas nas consultas de pré-natal. Todas as informações devem ser registradas no cartão da gestante e no prontuário das mesmas em todas as consultas. No caso de identificar fatores de risco, o profissional de saúde precisa ficar alerta e a gestante precisa ser referenciada para a atenção especializada ${ }^{3}$.

Dentre as ações educativas realizadas nas ESFs, podemse citar os grupos de gestantes, que têm como objetivo complementar o atendimento realizado nas consultas, possibilitando a troca de experiências e conhecimentos, além de proporcionar a criação do vínculo entre profissionais e usuárias, diminuindo a ansiedade e esclarecendo as dúvidas das gestantes que surgem nesse período ${ }^{22}$.

Pr11 'Tem um grupo de gestante nas segundasfeiras de 15 em 15 dias. Quem participa é quase toda a equipe como a fonoaudióloga, a técnica [de enfermagem], enfermeira, nutricionista, educadora física, é praticamente toda a equipe. Elas têm uma agenda programada para as atividades'.

No grupo se desenvolvem atividades educativas e trocas de saberes, juntamente com equipe interdisciplinar, sendo visto como uma estratégia para melhoria da qualidade de vida das gestantes ${ }^{22}$. Ficou constatado em outro estudo, que a realização de ações educativas como grupo de gestantes, tem como objetivo fornecer informações e orientações, estabelecer vínculo profissional/paciente, favorecer a interação do paciente com a unidade, auxiliar na promoção e prevenção da saúde, além de compartilhar experiências/ vivências ${ }^{23}$.

Pr13 'A gente retornou com o grupo de gestante, estava um pouco parado, são mensais. Nós chamamos e perguntamos as dúvidas delas (...) nos trouxeram que querem saber sobre o parto humanizado (...)'. 
Pr20 '(...) fazem o grupo de gestantes, eu só não sei se é quinzenal, (...), mas, eu sei que é bem no dia da consulta que elas têm com o médico, então daí elas vem $(\ldots)^{\prime}$.

As gestantes que participam de grupos educativos sentem-se mais seguras no período pós-parto, tanto nos cuidados com o recém-nascido, quanto consigo mesmas. As gestantes também relataram que esse tipo de ação educativa contribui para sanar as dúvidas, diminuindo os medos e anseios durante o processo gestacional e no momento do parto ${ }^{24}$. Por outro lado, alguns profissionais do presente estudo relataram que não há grupo de gestantes nas ESFs em que atuam.

Pr3 '(...) grupo de gestante não temos. (...) o que sim é a consulta mais prolongada. Como a gente tem pouca demanda, a gente não faz a consulta de vinte minutos restritos como tem que ser, a gente pode conversar mais, pode estar mais à vontade, orientar, explicar, então acho que essa atividade de prolongar consulta já supre a necessidade de um grupo'.

Pr25 'Se a gente convidar as nossas não vem nenhuma aqui (...). O certo seria um grupo porque geralmente elas têm as mesmas dúvidas, mas nós temos essa particularidade aqui de ser pouca gestante (...)'.

Alguns profissionais relataram que não há grupo de gestantes, havendo apenas a consulta mais prolongada, sendo que um destes sabe da necessidade e da diferença de realizar grupo como ação educativa. Os grupos são revelados como recurso essencial, pois proporcionam o compartilhamento de experiências entre as gestantes, bem como dúvidas, angústias e saberes, auxiliando como construção de conhecimentos. Os grupos servem para evitar práticas fragmentadas que provocam distanciamento entre profissional e o paciente, garantindo a integralidade do cuidado e o acesso adequado e de qualidade aos serviços de saúde ${ }^{25}$.

A formação de grupos de gestantes é uma estratégia eficaz, que quando associada às consultas individuais, contribui para uma assistência de qualidade ${ }^{26}$.

Os profissionais têm adotado estratégias para implementar o grupo de gestantes nas ESFs para incorporar como ações na assistência pré-natal.

Pr7 'O grupo, eu fiz duas tentativas, uma eu disse que era consulta, fiz o agendamento, elas compareceram todas, achando que ia ser a consulta tradicional e era o grupo, daí deu quórum e a outra que daí eu avisei que iria ser o grupo, não veio ninguém'.
Pr12 '(...) como tinha pouca aderência nos grupos (...) decidiu-se fazer um grupo antes das consultas, meia hora antes (...) passa vídeos educativos, conversa sobre as dúvidas, serve um chazinho. (...) sorteia brindes com as doações que recebe, depois elas ficam trocando experiências umas com as outras, é bem legal (...)'.

O grupo realizado antes das consultas de pré-natal mostrou-se como uma estratégia eficaz, visto que as gestantes participam e após são encaminhadas para a consulta individual, possibilitando às gestantes refletir e trocar experiências sobre o período gravídico e puerperal ${ }^{4}$. A consulta individual associada aos grupos, contribui para a expansão da cobertura pré-natal, garantindo uma assistência integral e humanizada às mulheres ${ }^{27}$.

Foi referido pelos profissionais deste estudo que 7 ESFs realizam os grupos de gestantes como uma estratégia de educação em saúde e 6 ESFs não realizam, devido à pouca demanda de gestantes, ou ao não comparecimento de gestantes apenas para os grupos, ou até mesmo por não haver ACS em todo o território de abrangência.

Destaca-se a importância de ações multiprofissionais para implementar dispositivos nos grupos de gestantes nas ESFs como hábito de encontros mensais, nos casos em que há poucas gestantes, ou ainda antes das consultas individuais para uma assistência pré-natal qualificada.

\section{Rotinas e limites na realização de exames e procedimentos obstétricos}

Um dos dez passos para o pré-natal de qualidade na atenção básica refere-se a solicitação, realização e avaliação em tempo oportuno do resultado dos exames preconizados no atendimento pré-natal ${ }^{8}$. Assim, salienta-se a realização destes exames para detecção e tratamento precoce de doenças.

Pr2 'É realizar o teste rápido de HIV e sífilis que nos disponibilizam e pedir os exames do primeiro trimestre (...), todos os exames preconizados no pré-natal de baixo risco, então, hemograma, glicose, $H I V, V D R L, E Q U$, tipagem sanguínea e também é realizado o cadastro da gestante no SIS pré-natal'.

Constatou-se que os profissionais das ESFs, solicitam os exames conforme protocolo do MS logo após o diagnóstico da gravidez, na primeira consulta ou quando a gestante é acolhida na unidade ${ }^{8}$.

No que concerne às dificuldades encontradas pelos profissionais, observou-se uma grande queixa quanto à demora na realização da ultrassonografia obstétrica pelo Sistema Único de Saúde (SUS). 
Pr5 'O ultrassom é um caso de toda a rede pública que demora bastante tempo e por vezes solicita-se o transvaginal, mas está no final do primeiro trimestre, já solicita o morfológico para dar tempo de (...) fazer na idade adequada'.

Pr8 'Demora (...) uns trinta dias. Às vezes não dá tempo de fazer o ultrassom da translucência nucal, no dia que ela tem que fazer. (...) o ultrassom morfológico também nunca dá certo, aí geralmente elas pagam (...)'.

Recomenda-se que a ultrassonografia de rotina durante a gestação seja solicitada para melhor determinar a idade gestacional, para auxiliar na detecção precoce de gestações múltiplas e nas malformações fetais. Precisa ser realizada no primeiro trimestre gestacional (entre 10 e 13 semanas), para determinar a medida da translucência nucal, a fim de investigar anomalias ${ }^{28,7}$.

A prática do exame ultrassonográfico no sistema público é um fator preocupante no cuidado pré-natal sob o ponto de vista dos profissionais, devido à demora que existe na regulação da secretaria de saúde do município de Santa Maria/RS, a principal causa para algumas gestantes realizar o ultrassom em clínicas da rede de saúde suplementar.

Pr6 '(...) como demora! Muitas acabam pegando um pedido de ultrassom e fazendo em alguma clínica, para agilizar (...' .

Pr23 'O ultrassom, faz-se o pedido e elas agendam ali na frente (secretaria da ESF). (...) como é pelo SUS demora mais até ser pedido (...), mas geralmente a maioria opta por fazer particular (...)'.

Enfermeiros relataram a grande demora dos resultados dos exames solicitados nas consultas de pré-natal, podendo esses resultados demoraram até três meses para chegar, tornando-se um problema devido a identificação tardia de alguma complicação, que poderia ter sido detectada e tratada precocemente se estes resultados estivessem prontos em tempo oportuno. Também mencionaram que muitas vezes as gestantes precisam realizar o ultrassom em clínicas da rede suplementar, para receber o resultado imediato, corroborando com as falas dos profissionais deste estudo ${ }^{29}$.

\section{Atuação multiprofissional e seus diferentes papéis no atendimento Pré-Natal}

A assistência pré-natal e puerperal precisa ser acompanhada por uma equipe multiprofissional, sendo que as consultas podem ser feitas tanto por médico quanto por enfermeiro conforme o Decreto no 94.406/87. A equipe mínima das ESFs preconizado pelo MS é composta por médico, enfermeiro, técnico em enfermagem e agente comunitário de saúde ${ }^{8}$.

O Técnico em Enfermagem têm papel crucial na assistência pré-natal no que diz respeito à realização de ações educativas a fim de contribuir para a saúde materna e infantil.

Pr1 '(...) passa por mim, verifico sinais (vitais), converso um pouquinho e anoto na ficha de atendimento alguma observação e as minhas percepções também (...)'.

Pr9 'Eu verifico os sinais [vitais] quando elas chegam, todas as consultas se verifica pressão arterial, se tem problema de diabetes, (...) faz o HGT [hemoglucoteste], mas em princípio é peso e altura (...) que vai para o cadastro $(\ldots)^{\prime}$.

É atribuição do Técnico em Enfermagem realizar ações educativas para as mulheres e suas famílias; aferir o peso, a altura e a pressão arterial e registrar os dados no cartão da gestante; fornecer medicação, mediante receita médica ou medicamentos padronizados para o programa; aplicar vacina antitetânica e participar das atividades educativas ${ }^{3}$.

Além disso, foi observado em uma fala, que o profissional acima citado não considera suas ações prestadas à gestante como parte da consulta pré-natal, referindo que a consulta é realizada pela Enfermagem.

Pr20 'Mais ou menos, porque na verdade ali o que eu mais faço é pesar e passar pra elas [Enfermeiras]. A gente tem que pesar, verificar a pressão, depois o resto fica tudo com elas [Enfermeiras] essa parte de papel, eu não tenho muito contato'.

Os Agentes Comunitários de Saúde (ACS) que integram as equipes das ESFs exercem elo de comunicação e integração da população com os serviços de saúde, tendo conhecimentos, habilidades e competências específicas na saúde ${ }^{30}$. Quanto à atenção ao pré-natal, os ACS têm o compromisso de identificar gestantes no território e orientá-las para um adequado acompanhamento pré-natal e puerperal.

Pr17 'O meu papel é orientação quanto à alimentação, às vacinas, em relação ao corpo; orientação quanto ao bebê, o cuidado com o umbigo (...), mas isso tudo segue um cronograma (...)'.

Pr22 'Nós enquanto agente comunitário de saúde, capta a gestante na comunidade e geralmente quem descobre primeiro, agendamos a primeira consulta na unidade, e se diagnosticado alguma alteração, encaminha-se para o hospital'. 
Compete ao ACS realizar visitas domiciliares, encaminhar a gestante ao serviço de saúde, realizar orientações necessárias para as consultas, identificar situações de risco e encaminhá-la ao serviço de saúde, realizar a captação precoce da gestante para a primeira e demais consultas, e orientação sobre planejamento familiar ${ }^{3,8}$.

O profissional médico deve compor a equipe das ESFs e prestar assistência pré-natal e puerperal, em que Ihe compete realizar anamnese e exame clínico-obstétrico da gestante; solicitar os exames necessários para cada trimestre gestacional; preencher o cartão da gestante e atualizá-lo a cada consulta; orientar quanto aos fatores de risco e sobre o tratamento se necessário; identificar as gestantes de risco e encaminhá-las para a unidade de referência; participar de grupos de gestantes e realizar visitas domiciliares, além de realizar consulta de pré-natal, intercalando com a do enfermeiro ${ }^{3,8}$.

Pr5 '(...) faço a ausculta dos batimentos, meço a altura uterina, pergunto das queixas com relação a sangramento, queixas de infecção urinária que predispõe a trabalho de parto prematuro; uma consulta obstétrica que possa ser feita por um clínico. E são solicitados os exames, aferido a pressão, pesagem pra ver o acompanhamento mensal, desde o início'.

Pr23 'A gestante chega aqui [ESF] converso com ela para ver se tem alguma queixa, se teve contração ou alguma intercorrência, (...) faço o exame físico, peso, pressão, ausculto $B C F$, dinâmica, movimentação fetal e preenche os dados na carteirinha. Se tem alguma gestante de alto risco encaminha para o pré-natal de alto risco [hospital] e solicita os exames de rotina'.

Por fim, com relação ao papel de atuação do enfermeiro no acompanhamento pré-natal, o mesmo pode prestar assistência integral à saúde da mulher, durante todo período gravídico e puerperal, sendo este um dos agentes de educação em saúde, realizando ações de promoção e práticas assistenciais à mulher e à criança ${ }^{3}$.

Pr21 No momento que identifica que deu positivo [para gravidez], fizemos o cadastro no Sisweb e se pede todos os primeiros exames do pré-natal laboratoriais, coleta o teste rápido de HIV e sífilis. Se o esposo estiver junto, tenta-se fazer também. Fizemos as primeiras orientações, preenchemos a carteira da gestante e realizamos a avaliação física da gestante (...)'.

Pr24 'Geralmente elas vão com queixa de atraso menstrual e se faz o teste rápido [gravidez] que tem na unidade. Se positivo, já faz o cadastro, agora está mais fácil por causa do sistema, sai até o número do SIS pré-natal (...)'.
Cabe ao enfermeiro orientar sobre o acompanhamento pré-natal; realizar o cadastro das gestantes no programa SIS Pré-natal; realizar consulta de pré-natal nas gestantes de baixo risco; identificar as gestantes de risco e encaminhálas ao serviço de alto risco; atualizar o cartão da gestante a cada consulta; realizar os testes rápidos e solicitar exames complementares; realizar exame clínico e obstétrico; prescrever medicamentos padrões; orientar quanto à vacinação, aleitamento materno, parto e puerpério; realizar atividades de educação em saúde individuais e em grupos de gestantes ou sala de espera; orientar sobre a frequência das consultas e realizar busca ativa das gestantes faltosas; realizar visitas domiciliares e intercalar as consultas com à do médico ${ }^{3,8}$.

Um fator relevante mencionado pelos enfermeiros é a questão do revezamento de consultas pré-natal entre médico e enfermeiro. O sistema de intercalar as consultas entre os dois profissionais está descrito no protocolo do MS nas ações tanto do enfermeiro quanto do médico 3 .

Pr26 'Combinei com a médica para a gente fazer assim: um mês comigo, o outro mês com ela [gestante] ser atendida por dois profissionais, enfermeiro e médico $(\ldots)^{\prime}$.

No presente estudo, 17 profissionais relataram que as consultas de pré-natal são realizadas intercaladas pelos médicos e enfermeiros. Já em outras três entrevistas, os profissionais mencionaram que somente o médico realizava as consultas e em apenas uma entrevista somente a enfermeira prestava assistência no pré-natal.

Outro aspecto observado em uma fala refere-se ao vínculo profissional-paciente, em que as gestantes comparecem mais nas consultas de enfermagem do que nas consultas médicas.

Pr24 '(...) A gente tenta fazer um dia com a enfermeira, aí na próxima consulta com o médico. (...) elas preferem até o pré-natal com a enfermeira, do que com o médico por causa da vinculação, até faltam às vezes quando é com o médico, daí quando é com a enfermeira elas comparecem'.

O vínculo estabelecido entre profissional-paciente é uma ferramenta essencial na busca da melhoria da qualidade da atenção à saúde e na integralidade do cuidado. O vínculo permite a aproximação do profissional com o paciente, possibilitando que as gestantes se sintam acolhidas e para que haja momentos de trocas, escuta qualificada e respeito ${ }^{31}$.

Em relação à atuação de cada profissional de saúde, observou-se que todos profissionais entrevistados seguem o protocolo do MS, que preconiza uma rotina de atendimento prestado durante o período pré-natal e puerperal nas ESF(s) para a realização das ações ${ }^{3,8}$. 


\section{Encaminhamento das gestantes para os serviços de referência e contra referência}

O sistema de referência e contra referência na saúde pública corresponde a uma rede de atenção integrada e hierarquizada entre os três níveis de complexidade, de modo crescente. A atenção integral assegura ao usuário o acesso aos serviços de saúde nos diferentes níveis, garantindo um fluxo ordenado na rede, contribuindo para a continuidade do cuidado e resolubilidade dos problemas ${ }^{32}$.

Para o adequado acompanhamento pré-natal os profissionais de saúde deverão avaliar os fatores de risco gestacional, a fim de identificá-los e tratá-los, dependendo do problema instalado, evitando um resultado desfavorável ${ }^{33}$. A Rede Cegonha orienta o fluxo que a gestante deve seguir no território, desde o diagnóstico da gestação até o momento do parto, onde deve ser classificado o risco e efetuado o encaminhamento da mesma ${ }^{34}$.

Quando se avalia e diagnostica o risco, a gestante precisa ser referenciada para um serviço especializado, e após a avaliação da conduta, a mesma deve ser contra referenciada para a atenção primária, com as recomendações da continuidade na assistência pré-natal e/ou permanecer o acompanhamento nos serviços de pré-natal de alto risco ${ }^{8}$.

Nos casos em que a gestante é contra referenciada para a atenção primária e mantém o acompanhamento nos dois serviços de referência, os profissionais na atenção primária, precisam seguir as orientações fornecidas pelo serviço de referência ${ }^{3}$. Neste estudo, questionou-se como se acontece os encaminhamentos aos serviços de referência.

Pr2 'É numa ficha de referência e contra referência, mas a contra referência nunca volta'.

Pr3 '(...) todos esses encaminhamentos se fazem com protocolo de encaminhamento que é através da secretaria municipal de saúde, com um formulário que a gente preenche de referência e contra referência. Aí indica a informação do paciente, um breve resumo da história clínica, os exames realizados, o possível diagnóstico e para quem se está encaminhando'.

Pr14 'O do serviço de referência faz-se via e-mail, envia e-mail para o setor de regulação deles e eles retornam com a data agendada (...)'.

Para concretizar o princípio da integralidade do cuidado, é necessário buscar meios para facilitar o sistema de referência e contra referência na rede de atenção. Estabelecer um protocolo para esse sistema é uma estratégia eficaz para garantir um adequado tratamento e acompanhamento prénatal ${ }^{32}$.
Fatores limitantes foram mencionados pelos profissionais no momento de encaminhar as gestantes aos serviços de referência.

Pr23 'Sempre que eu encaminho eu oriento - mesmo que tu continues, que fará o pré-natal no hospital, tu continuas vindo aqui na unidade - porque o território dela é aqui, para ter um acompanhamento, mas, elas não voltam, elas ficam só com o acompanhamento lá [hospital] (...). Escuta-se dizer é que lá [hospital] eles orientam elas a ficar só lá [hospital], aí elas não têm mais o acompanhamento no posto (...)'.

Segundo a Portaria № 1.020 de 29 de maio de 2013, quando a gestante é encaminhada para o acompanhamento no pré-natal de alto risco, a mesma precisa ser orientada a não perder o vínculo com a equipe da atenção básica que iniciou o seu pré-natal. Assim como, o serviço de pré-natal de alto risco mantenha a equipe da atenção básica informada sobre a evolução da gestação e dos cuidados à gestante que foi encaminhada. O profissional de saúde, portanto, é responsável por garantir à gestante o acompanhamento pré-natal nos dois níveis de atenção à saúde ${ }^{35}$.

Outro entrave mencionado pelos profissionais diz respeito à ausência de contra referência pelo serviço especializado para a atenção primária.

Pr6 '(...) é bem falho e não só na questão da gestante, referência e contra referência, (...) o problema de saúde de forma geral no município de Santa Maria, porque nenhum serviço se conversa com o outro, não tem esse diálogo (...). O que eu observo é que tem reclamações do hospital para atenção básica e tem nossa para o hospital, (...) fica um jogo, um empurra-empurra e não se consegue avançar nesse sentido'.

É essencial que haja integração entre os diferentes níveis de complexidade dos serviços de saúde, pois as informações referentes às gestantes auxiliam na assistência prestada pelos profissionais em outro nível de atenção. As informações normalmente não são repassadas para o profissional que irá prestar assistência, levando à fragmentação da atenção, fragilizando todas as ações realizadas até o nascimento ${ }^{2}$.

Neste sentido, observa-se por meio da análise das categorias elencadas que a atenção ao pré-natal necessita ser reorganizada. As estratégias centradas nas tecnologias leves (acolhimento, humanização, vínculo) foram relatas pelos profissionais como sendo desenvolvidas de forma satisfatória nas ESFs, enquanto que foram observados desafios no sistema de regulação (exames, referência e contra referência). Pôde-se perceber que o fluxo da gestante no território está 
bem estabelecido, entretanto, há ainda limitações entre os pontos de atenção na rede de cuidados à saúde.

\section{CONSIDERAÇÕES FINAIS}

O estudo reforça os achados de outros manifestados pelo olhar dos profissionais da saúde acerca da qualidade da assistência pré-natal nas ESFs do município de Santa Maria, RS. Destaca-se que todos os profissionais de saúde ao conduzirem as consultas de pré-natal fazem uso das recomendações preconizadas pelo Ministério da Saúde. Em contraponto, há fatores limitantes em relação à demora na realização da ultrassonografia obstétrica pelo SUS, levando algumas gestantes a optarem por fazer em clínicas da rede suplementar, bem como no que diz respeito à ausência de contra referência pelo serviço especializado para a atenção primária. Também foi notado à ausência de grupos de gestantes em algumas unidades de saúde em que estão inseridos, devido a não adesão das usuárias ou por não haver profissionais suficientes para a realização destes.

Conclui-se que há necessidade de repensar os processos de trabalho entre a Secretaria de Saúde do município e a atenção especializada na assistência pré-natal, para a elaboração de estratégias eficazes a fim de evitar práticas fragmentadas que provocam distanciamento entre profissional e o paciente, garantindo a integralidade do cuidado em todos os níveis de atenção e o acesso adequado e de qualidade e aos serviços de saúde, com o objetivo de reduzir as complicações gestacionais e auxiliar no desfecho do nascimento, bem como no acompanhamento puerperal.

Além disso, ressalta-se aqui a importância da continuidade da pesquisa, no que se refere à efetividade das estratégias de intervenção, além de analisar como está delineada a qualidade da assistência pré-natal em todas as Unidades Básicas de Saúde (UBS) do município, com o intuito de contribuir para a saúde materno-infantil.

\section{REFERÊNCIAS}

1. Coutinho T, Teixeira MTB, Dain S, Sayd JD, Coutinho LM. Adequação do Processo de Assistência Pré-natal entre as Usuárias do Sistema Único de Saúde em Juiz de Fora-MG. Rev Bras Ginecol Obstet. 2003;25(10):717-24. https://doi.org/10.1590/S010072032003001000004

2. Trevisan MR, Lorenzi DRS, Araújo NM, Ésber K. Perfil da assistência pré-natal entre usuárias do Sistema Único de Saúde em Caxias do Sul. Rev Bras Ginecol Obstet. 2002;24(5):293-9. https://doi. org/10.1590/S0100-72032002000500002

3. Ministério da Saúde (BR). Pré-natal e puerpério: atenção qualificada e humanizada. Brasília: Ministério da Saúde; 2006.

4. Ximenes Neto FRS. Qualidade da atenção ao pré-natal na ESF em Sobral - Ceará. Rev Bras Enferm. 2008;61(5):592-602. https://doi. org/10.1590/S0034-71672008000500011
5. Andrade ACV, Schwaim MT, Ceretta LB, Dagostin VS, Soratto MT. Planejamento das ações educativas pela equipe multiprofissional da Estratégia Saúde da Família. Mundo Saúde 2013;37(4):439-49. https://doi.org/10.15343/0104-7809.2013374439449

6. Carvalho DSC, Novaes HMD. Avaliação da implantação de programa de atenção pré-natal no Município de Curitiba, Paraná, Brasil: estudo em coorte de primigestas. Cad Saude Publica. 2004;20 Suppl 2:S220-30. https://doi.org/10.1590/S0102-311X2004000800017

7. Cunha MA, Dotto LMG, Mamede MV, Mamede FV. Assistência prénatal: competências essenciais desempenhadas por enfermeiros. Esc Anna Nery Rev Enferm. 2009;13(1):145-53. https://doi. org/10.1590/S1414-81452009000100020

8. Ministério da Saúde (BR). Atenção ao pré-natal de baixo risco. Brasília: Ministério da Saúde; 2012.

9. Ministério da Saúde (BR). Resolução no 466, de 12 de dezembro de 2012. Brasília: Ministério da Saúde; 2012.

10. Minayo MCS. O desafio do conhecimento. 10ạ ed. São Paulo: Hucitec; 2007.

11. Minayo MCS. O desafio do conhecimento: pesquisa qualitativa em saúde. 12a ed. São Paulo: Hucitec-Abrasco; 2013.

12. Cavalcante RB, Calixto P, Pinheiro MMK. Análise de conteúdo: considerações gerais, relações com a pergunta de pesquisa, possibilidades e limitações do método. Inf \& Soc Est. 2014;24(1): 13-8.

13. Ministério da Saúde (BR). Programa humanização do parto: humanização no pré-natal e nascimento. Brasília: Ministério da Saúde; 2002.

14. Castro AJR, Shimazaki, ME. Protocolos clínicos para unidades básicas de saúde. Belo Horizonte: Escola de Saúde Pública; 2006.

15. Solla JJSP. Acolhimento no sistema municipal de saúde. Rev Bras Saúde Matern Infant. 2005;5(4):493-503. https://doi.org/10.1590/ S1519-38292005000400013

16. Ministério da Saúde (BR). Acolhimento à demanda espontânea. Brasília: Ministério da Saúde; 2013.

17. Gonçalvez R, Urasaki MBM, Merighi MAB, D'ávila CG. A avaliação da efetividade da assistência pré-natal de uma unidade de saúde da familia em um município da grande São Paulo. Rev Bras Enferm. 2008;61(3):349-53.

18. Ceccim RB. Educação permanente em saúde: desafio ambicioso e necessário. Interface - Comunic, Saúde, Educ. 2005;9(16):161-77. https://doi.org/10.1590/S1414-32832005000100013

19. Ministério da Saúde (BR). Portaria no 1.459, de 24 de Junho de 2011. Brasília: Ministério da Saúde; 2011.

20. Beningna MJC, Nascimento WG, Martins JL. Pré-natal no Programa de Saúde da Família (PSF): com a palavra, os enfermeiros. Cogitare Enferm. 2004;9(2):23-31.

21. Landerdahl MC, Cabral FB, Ressel LB, Gonçalves MO, Martins FB. A percepção de mulheres sobre atenção pré-natal em uma unidade básica de saúde. Esc Anna Nery R Enferm. 2007;11(1):105-11. https://doi.org/10.1590/S1414-81452007000100015

22. Frigo LF, Silva RM, Mattos KM, Manfio F, Boeira GS. A importância dos grupos de gestante na atenção primária: um relato de experiência. Rev Epidemiol Control Infect. 2012;2(3):113-4. https:// doi.org/10.17058/reci.v2i3.2745 
23. Camargo AM, Silva APBV, Wolff LDG, Soares VMN, Gonçalves CGO. Abordagens grupais em saúde coletiva: a visão de usuários e de profissionais de enfermagem. Rev Atenção Saúde. 2012;10(31):1-9. https://doi.org/10.13037/rbcs.vol10n31.1475

24. Souza VB, Roecker S, Marcon SS. Ações educativas durante a assistência pré-natal: percepção de gestantes atendidas na rede básica de Maringá-PR. Rev Eletr Enf. 2011;13(2):199-210. https:// doi.org/10.5216/ree.v13i2.10162

25. Vieira MS. Grupo de gestantes na Equipe Saúde da Família: proposta de implantação no Centro de Saúde Confisco, Belo Horizonte, Minas Gerais [trabalho de conclusão de curso]. Belo Horizonte: Universidade Federal de Minas Gerais; 2011.

26. Vasconcelos CTM, Machado MFAS, Becker SLM. Educação em saúde a gestantes utilizando a estratégia grupo. Rev RENE. 2007;8(3):107-16

27. Penna LHG, Carinhanha JI, Rodrigues RF. Consulta coletiva de prénatal: uma nova proposta para uma assistência integral. Rev Latinoam Enfermagem. 2008;16(1):158-60. https://doi.org/10.1590/ S0104-11692008000100024

28. Ministério da Saúde (BR). Política Nacional de Atenção Básica. Brasília: Ministério da Saúde; 2012.
29. Guerreiro EM, Rodrigues DP, Silveira MAM, Lucena NBFL. O cuidado pré-natal na atenção básica de saúde sob o olhar de gestantes e enfermeiros. REME - Rev Min Enferm. 2012;16(3):315-23.

30. Duncan BB, Schmidt MI, Giugliani ERJ. Medicina ambulatorial: condutas em atenção primária à saúde baseadas em evidências. $3^{\text {a }}$ ed. Porto Alegre: Artmed; 2004.

31. Brunello MEF, Ponce MAZ, Assis EG, Andrade RLP, Scatena LM, Palha PF, Villa TCS. O vínculo na atenção à saúde: revisão sistematizada na literatura, Brasil (1998-2007). Acta Paul Enferm. 2010;23(1):131-5. https://doi.org/10.1590/S0103-21002010000100021

32. Dias CF. O sistema de referência e contrarreferência na estratégia saúde da família no município de Bauru: perspectiva dos gestores [dissertação]. Botucatu: Universidade do Estado de São Paulo; 2010.

33. Ministério da Saúde (BR). Gestação de alto risco: manual técnico. Brasília: Ministério da Saúde; 2010.

34. Universidade Federal do Maranhão; Universidade Aberta do SUS. Redes de atenção à saúde: a Rede Cegonha. São Luís: UFMA/UNASUS; 2016

35. Ministério da Saúde (BR). Portaria no 1.020, de 29 de maio de 2013. Brasília: Ministério da Saúde; 2013. 